\title{
Solution of the Burger's Equation for Longitudinal Dispersion Phenomena Occurring in Miscible Phase Flow through Porous Media
}

\author{
Mitesh S. Joshi ${ }^{1 *}$, Narendrasinh B. Desai ${ }^{2}$ \& Monika N. Mehta ${ }^{3}$ \\ ${ }^{1}$ Department of Mathematics, C.K. Pithawala College of Engineering and Technology, \\ Surat-395 007, India \\ 2 Department of Mathematics, A.D.Patel Institute of Technology, \\ New V.V.Nagar -388121, India \\ ${ }^{3}$ Professor Department of Mathematics, S.V. National Institute of \\ Technology, Surat- 395 007, India \\ Email: mitesh joshi2003@yahoo.com
}

\begin{abstract}
An approximate solution of longitudinal dispersion phenomena occurring in two phase miscible fluid flow through porous media has been obtained by using the group theoretic approach. The longitudinal dispersion coefficient is assumed to be directly proportional to the concentration of the fluid for a distance $\mathrm{x}$ and at any time $t>0$. The graphical representation for the concentration of the fluid for a distance $\mathrm{x}$ and at time $t>0$ has been obtained using Mat lab coding.
\end{abstract}

Keywords: Burger's equation; longitudinal dispersion; Riccati equation.

\section{Introduction}

The present paper discusses the problem of longitudinal dispersion, which occurs during two-phase flow of miscible fluids through porous media. The governing equation non-linear partial differential equation yields into the form of Burger's equation and its solution has been obtained by using Group theoretic approach. In saturated flow through porous media, velocities vary widely across any single pore, just as a capillary tube where the velocity distribution in laminar flow is parabolic. In addition, the pores possess different sizes, shapes, and orientations. As a result when a labeled miscible liquid, referred as a tracer, is introduced into a flow system, it spreads gradually to occupy an increasing portion of the flow region. This phenomenon is known as dispersion and constitutes a non-steady, irreversible mixing process by which the tracer disperses within the surrounding fluid.

Dispersion is essentially a microscopic phenomenon caused by a combination of molecular diffusion and hydrodynamic mixing occurring with laminar flow through porous media. The net result produces a conic downstream form, from a continuous point source. This phenomenon can be observed in coastal areas, Received October $14^{\text {th }}, 2010$, Revised May $12^{\text {th }}, 2011$, Accepted for publication October $25^{\text {th }}, 2011$. Copyright (C) 2012 Published by LPPM ITB \& PII, ISSN: 1978-3051, DOI: 10.5614/itbj.eng.sci.2012.44.1.5 
where the fresh waterbeds are gradually displaced by seawater. This phenomenon plays an important role in the seawater intrusion into reservoir at rive mouths and in the underground recharge of wastewater. The hydrodynamic dispersion is the macroscopic outcome of the actually movement of individual tracer particles through the pores. Various physical and chemical phenomena occur simultaneously due to molecular diffusion and convection. These day's efforts are being made by the environmentalist to dispose the atomic waste products born from nuclear reactor and dumped inside the ground by using the same phenomenon of displacement. Miscible displacement in porous media plays a prominent role in many engineering and scientific fields such as oil recovery in petroleum engineering, contamination of ground water by waste products disposed underground movement of minerals in the soil and recovery of spent liquors in pulping process.

These problems of dispersion have been receiving considerable attention from chemical, environmental and petroleum engineers, hydrologists, mathematicians and soil scientists. Most of the works reveals common assumption of homogenous porous media with constant porosity, steady seepage flow velocity and constant dispersion coefficient. For such assumptions, Ebach and White [1] studied the longitudinal dispersion problem for an input concentration that varies periodically with time and Ogata and Banks [2] for a constant input concentration. Hoopes and Herteman [3] studied the problem of dispersion in radial flow from a well fully penetrating, homogenous, isotropic non-adsorbing confined aquifers. Bruce and Street [4] considered both longitudinal and lateral dispersion in semi-infinite non adsorbing porous media in a steady unidirectional fluid flow for a constant input concentration. Marino [5] considered the input concentration varying exponentially with time. Al-Niami and Rushton [6] and Marino [5] studied the analysis of flow against dispersion in a porous media. Basak [7] presents an analytical solution the problem of evaporation from a horizontal soil column in which diffusivity increases linearly with moisture content and also to a problem of concentration dependent diffusion with decreasing concentration at the source. Hunt [8] applied perturbation method to longitudinal and lateral dispersion in non uniform seepage flow through heterogeneous aquifers. Wang [9] discussed the concentration distribution of a pollutant arising from a instantaneous point source in a two dimensional water channel with non uniform velocity distribution. He employed Gill's method to solve the convective diffusion equation. Kumar [10] discussed the dispersion of pollutants in semi-infinite porous media with unsteady velocity distribution. Mehta and Patel [11] applied Hope-Cole transformation to unsteady flow against dispersion of miscible fluid flow through porous media. Mehta and Saroj [12] considered that the longitudinal dispersion coefficient is directly proportional to the concentration 
and the velocity component in direction of $X$-axis is considered directly proportional to ratio of distance $x$ and time t.

One dimensional treatment of dispersion phenomena avoids the treatment of radial or transverse component of dispersion. The mixture of fluids under condition of complete miscibility could be thought to behave as a single phase fluid. Hence it will obey the Darcy's law. The present paper discuss the analytical solution of nonlinear partial differential equation for longitudinal which takes place when miscible fluids mixes in the direction of flow. The mathematical formulation of the problem yields into Burger's equation and its solution is obtained by Group theoretic approach. Group theoretic methods provide a powerful tool because they are not based on linear operators, superposition, or any other aspects of linear solution techniques. Therefore, these methods are applicable to non-linear differential models.

\section{Statement of the Problem}

Miscible displacement in porous media is a type of double-phase flow in which the two phases are completely soluble in each other. Therefore, capillary forces between the two fluids do not come into effect. At first it must be thought that miscible displacement could be described in a very simple fashion. The mixture under conditions of complete miscibility could be thought to behave, locally at least, as a single-phase fluid, which would obey Darcy's law. The change of concentration, in turn, would be caused by diffusion along the flow channels and thus be governed by the bulk coefficients of diffusion of the one fluid in the other. In this fashion, one arrives at a heuristic description of miscible displacement, which looks, at a first glance, at least very plausible.

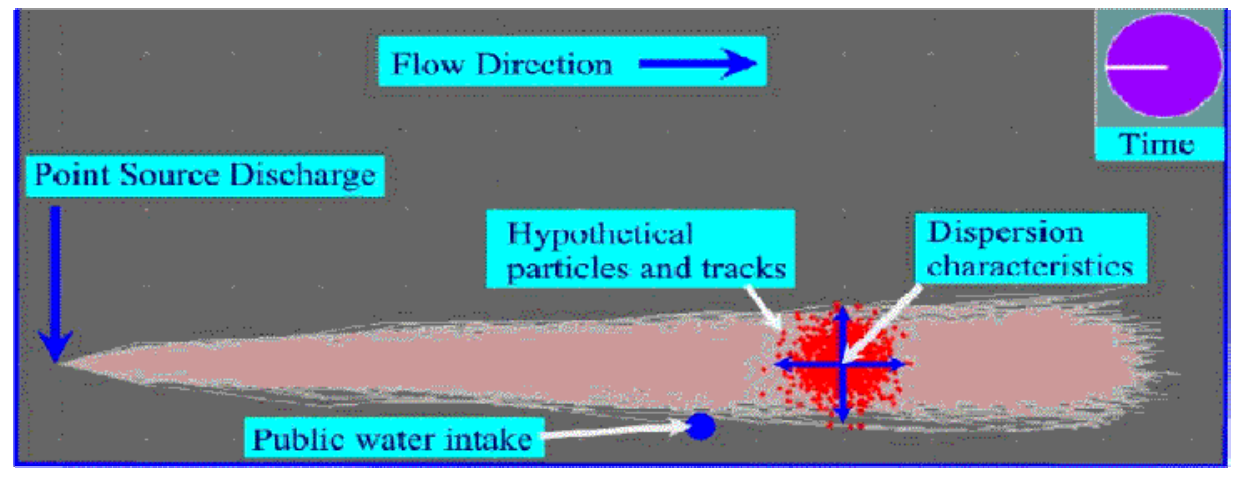

Figure 1 Longitudinal and Transverse dispersion.

The problem is to describe the growth of the mixed region, i.e. to find concentration as a function of time $t$ and position $x$, as the two miscible fluids 
flow through porous media. Outside of the mixed zone (on either side) the single-fluid equations describe the motion. The problem is more complicated, even in one dimension with fluids of equal properties, since the mixing takes place both longitudinally and transversely. Imagine at $t=0$ we inject a 'dot' of traced fluid of concentration $\mathrm{c}_{0}$ mass / length ${ }^{-3}$ rather than over the entire face. This situation is sketched in the following (Fig. 1). As the dot moves from left to right it will spread in the direction of flow and perpendicular to the flow. At the right the dot has transformed into an ellipsoid with concentration varying from $c_{0}$ to $c$ across it.

\section{$3 \quad$ Mathematical Formulation}

According to Darcy's law, the equation of continuity for the mixture, in the case of incompressible fluids is given by Bear [13].

$$
\frac{\partial \rho}{\partial t}+\operatorname{div} \cdot(\rho \bar{V})=0
$$

where, $\rho$ is the density for mixture and $\bar{V}$ is the pore seepage velocity vector.

The equation of diffusion for a fluid flow through a homogeneous porous medium, without increasing or decreasing the dispersing material is given by

$$
\frac{\partial C}{\partial t}+\operatorname{div}(C \bar{V})=\nabla \cdot\left[\rho \bar{D} \operatorname{div}\left(\frac{C}{\rho}\right)\right]
$$

Where $C$ is the concentration of the fluid $A$ into the other host fluid $B$ (i.e. $C$ is the mass of A per unit volume of the mixture) and $D$ of unit $\left[\right.$ length $^{2}$. time $\left.^{-1}\right]$ is the tensor coefficient of dispersion with nine components $D_{i j}$.

In a laminar flow through a homogeneous porous medium at constant temperature $\rho$ may be considered as constant. Then

$$
\operatorname{div} \cdot \bar{V}=0
$$

And hence Eq. (2) may be written as

$$
\frac{\partial C}{\partial t}+\bar{V} \cdot \nabla C=\operatorname{div}[\bar{D} \cdot \operatorname{div} C]
$$


When the seepage velocity $\bar{V}$ is along the x-axis, the non-zero components are $D_{11}=D_{L}=\frac{L}{c_{0}^{2}} \quad$ (coefficient of longitudinal dispersion) and $D_{22}=D$ (coefficient of transverse dispersion) and other $D_{i j}$ are zero. In this case the Eq. (4) becomes,

$$
\frac{\partial C}{\partial t}+u \frac{\partial C}{\partial x}=D_{L} \frac{\partial^{2} C}{\partial x^{2}}
$$

Where $\mathrm{u}$ is the component of velocity along the $\mathrm{X}$-axis having the dimension $\left[\right.$ length $\cdot$ time $\left.^{-1}\right]$ which is time dependent and $D_{L}>0$.it is the cross-sectional flow velocity of porous medium. Hence we have,

$$
u=\frac{C(x, t)}{c_{0}} \text { for } x>0
$$

Using the dimensionless variables $X=\frac{c_{0} x}{L}, T=\frac{t}{L}$, the Eq. (5), can be rewritten as,

$$
\frac{\partial C}{\partial T}+C \frac{\partial C}{\partial X}=\frac{\partial^{2} C}{\partial X^{2}}
$$

The boundary and initial conditions in longitudinal direction are given by

$$
\begin{aligned}
& C(X, 0)=\varepsilon ; \text { where } \varepsilon \text { is very small } \\
& C(0, T)=c_{0} q(T)(T>0) \\
& \lim _{X \rightarrow 1} C(X, T)=0(T>0)
\end{aligned}
$$

Thus Eq. (7) together with boundary conditions (8) to (10) represents the boundary value problem for the longitudinal dispersion of miscible fluid flow through a homogeneous porous medium. 


\section{$4 \quad$ Solution of the Problem}

Let

$$
C(X, T)=u(X, T) \cdot q(T)
$$

Where, $q(T)$ is an unknown function. Its proper form will be determined later. Thus differential Eq. (7) takes the form

$$
q(T) \frac{\partial u}{\partial T}+u \frac{d q}{d T}+q^{2} u \frac{\partial u}{\partial X}=q \frac{\partial^{2} u}{\partial X^{2}}, X>0, T>0
$$

and hence the initial and boundary conditions

$$
\begin{aligned}
& u(0, T)=c_{0}, \quad T>0, c_{0} \neq 0 \\
& \lim _{X \rightarrow 1} u(X, T)=0
\end{aligned}
$$

Our method depends on application of one-parameter group transformation to partial differential Eq. (7). Under this transformation two independent variables will be reduced by one and differential Eq. (7) transforms into ordinary differential equation.

\subsection{The Group Systematic Formulation}

The procedure is initiated with the group $\mathrm{G}$, a class of transformation of oneparameter $(a)$ of the form,

$$
\left.\begin{array}{l}
\bar{X}=M^{X}(a)+N^{X}(a) \\
\bar{T}=M^{T}(a)+N^{T}(a) \\
\bar{u}=M^{u}(a)+N^{u}(a) \\
\bar{q}=M^{q}(a)+N^{q}(a)
\end{array}\right\}
$$

Where, M's and N's are real valued and at least differentiable in real $\operatorname{argument}(a)$. 


\subsection{The Invariance Analysis}

To, transform the differential equation, transformation of the derivatives of $\mathrm{u}$ and $\mathrm{q}$ are obtained from $\mathrm{G}$ via chain-rule operations:

$$
\frac{\overline{\partial u}}{\partial X}=\left(\frac{M^{u}}{M^{X}}\right) \frac{\partial u}{\partial X} \text { and } \overline{\frac{\partial u}{\partial T}}=\left(\frac{M^{u}}{M^{T}}\right) \frac{\partial u}{\partial T}
$$

and

$$
\frac{\overline{d q}}{d T}=\left(\frac{M^{q}}{M^{T}}\right) \frac{d q}{d T}
$$

Eq. (12) is said to be invariantly transformed for some function $H(a)$, whenever

$$
\begin{aligned}
\bar{q} \frac{\partial \bar{u}}{\partial \bar{T}}+\bar{u} \frac{d \bar{q}}{d \bar{T}} & +\bar{q}^{2}-\frac{\partial \bar{u}}{\partial \bar{X}}-\bar{q} \frac{\partial^{2} \bar{u}}{\partial \bar{X}^{2}} \\
& =H(a)\left[q \frac{\partial u}{\partial T}+u \frac{d q}{d T}+q^{2} u \frac{\partial u}{\partial X}-q \frac{\partial^{2} u}{\partial X^{2}}\right]
\end{aligned}
$$

Substitution (17) into Eq. (17) yields,

$$
\begin{aligned}
\left(M^{q} q+N^{q}\right)\left(\frac{M^{u}}{M^{T}}\right) \frac{\partial u}{\partial T} & +\left(M^{u} u+N^{u}\right)\left(\frac{M^{q}}{M^{T}}\right) \frac{d q}{d T} \\
& +\left(M^{q} q+N^{q}\right)^{2}\left(M^{u} u+N^{u}\right)\left(\frac{M^{u}}{M^{Z}}\right) \frac{\partial u}{\partial X} \\
& -\left(M^{q} q+N^{q}\right)\left(\frac{M^{u}}{\left(M^{X}\right)^{2}}\right) \frac{\partial^{2} u}{\partial X^{2}} \\
& =H(a)\left[q \frac{\partial u}{\partial T}+u \frac{d q}{d T}+q^{2} u \frac{\partial u}{\partial X}-q \frac{\partial^{2} u}{\partial X^{2}}\right]
\end{aligned}
$$

From which 


$$
\begin{aligned}
& {\left[\frac{M^{q} M^{u}}{M^{T}}\right] q \frac{\partial u}{\partial X}+\left[\frac{M^{q} M^{u}}{M^{T}}\right] u \frac{d q}{d T}+\left[\frac{\left(M^{u} M^{q}\right)^{2}}{M^{X}}\right] u q^{2} \frac{\partial u}{\partial X} } \\
&-\left[\frac{M^{u} M^{q}}{\left(M^{X}\right)^{2}}\right] q \frac{\partial^{2} u}{\partial X^{2}}+R(a) \\
&=H(a)\left[q \frac{\partial u}{\partial T}+u \frac{d q}{d T}+q^{2} u \frac{\partial u}{\partial X}-q \frac{\partial^{2} u}{\partial X^{2}}\right]
\end{aligned}
$$

where,

$$
\begin{aligned}
R(a)= & {\left[\frac{N^{q} M^{u}}{M^{T}}\right] \frac{\partial u}{\partial T}+\left[\frac{M^{q} N^{u}}{M^{T}}\right] \frac{d q}{d T} } \\
& +\left[N^{u}\left(M^{q} q+N^{q}\right)^{2}\left(\frac{M^{u}}{M^{X}}\right)\right] u \frac{\partial u}{\partial X}-\left[\frac{M^{u} N^{q}}{\left(M^{X}\right)^{2}}\right] \frac{\partial^{2} u}{\partial X^{2}}
\end{aligned}
$$

The invariance of (19) implies $R(a) \equiv 0$. This is satisfied by putting

$$
N^{q}=N^{u}=0
$$

and hence we get,

$$
\left[\frac{M^{q} M^{u}}{M^{T}}\right]=\left[\frac{\left(M^{u} M^{q}\right)^{2}}{M^{X}}\right]=\left[\frac{M^{u} M^{q}}{\left(M^{X}\right)^{2}}\right]=H(a)
$$

Which yields,

$$
M^{T}=\left(M^{X}\right)^{2}=M^{q} M^{u}=\frac{1}{M^{X}}
$$

Moreover, boundary conditions (13) and (14) are also invariant in form, implying that

$$
N^{Z}=N^{u}=0 \text { and } M^{u}=1
$$

And the invariance of initial condition (8) implies that 


$$
N^{T}=0
$$

Finally, we get the one-parameter group $G$ which transforms invariantly differential Eq. (12), as well as initial condition (13) and boundary conditions (14). The group $\mathrm{G}$ is of the form

$$
G:\left\{\begin{array}{l}
\bar{X}=M^{X} X, \\
\bar{T}=\left(M^{X}\right)^{2} T, \\
\bar{u}=u \\
\bar{q}=q\left(\frac{1}{M^{X}}\right) q
\end{array}\right.
$$

\subsection{The Complete set of Absolute Invariant}

Our aim is to make use of the group methods to represent the problem in the form of an ordinary differential equation. Then we have to proceed in our analysis to obtain a complete set of absolute invariants.

If $\eta \equiv \eta(X, T)$ is the absolute invariant of the independent variables, then

$$
g_{j}(X, T ; u, q)=F_{j}[\eta(X, T)] \text { where } \mathrm{j}=1,2
$$

are two absolute invariants corresponding to $\mathrm{u}$ and $\mathrm{q}$. the application of a basic theorem in group theory, Moran [14] states that a function $g(X, T ; u, q)$ is an absolute invariant of a one-parameter group if it satisfies the following firstorder linear differential equation:

$$
\sum_{i=1}^{4}\left(\alpha_{i} f_{i}+\beta_{i}\right) \frac{\partial g_{i}}{\partial f_{i}}=0, \text { where } f_{i}=X, T, u, q
$$

where,

$$
\alpha_{i}=\frac{\partial M^{f_{i}}}{\partial a}\left(a^{0}\right) \text { and } \beta_{i}=\frac{\partial N^{f_{i}}}{\partial a}\left(a^{0}\right) ; \mathrm{i}=1,2,3,4
$$

and $a^{0}$ denotes the value of "a" which yields the identity element of the group. From which we get $\alpha_{3}=0$ and $\beta_{i}=1,2,3,4$ 
From Eq. (26), $\eta(Z, T)$ is an absolute invariant if it satisfies

$$
\alpha_{1} X \frac{\partial \eta}{\partial X}+\alpha_{2} t \frac{\partial \eta}{\partial T}=0
$$

Which, has a solution in the form

$$
\eta(X, T)=\frac{X}{T^{\beta}}, \beta=\frac{\alpha_{1}}{\alpha_{2}}>0
$$

By similar analysis the absolute invariants of the dependent variables $u$ and $q$ are $q(T)=\Gamma(T) \psi(\eta)$. Since, $q(T)$ and $\Gamma(T)$ are independent of $X$, while $\eta$ is a function $X$ and T, then $\psi(\eta)$ must be a constant, say $\psi(\eta)=1$, from which

$$
q(T)=\Gamma(T)
$$

and the second absolute invariant is

$$
u(X, T)=F(\eta)
$$

\subsection{Reduction to an Ordinary Differential Equation}

Substituting from (29) to (31) into Eq. (12), we get

$$
\frac{d^{2} F}{d \eta^{2}}+\left[\beta X T^{\beta-1}\right] \frac{d F}{d \eta}-\left[\Gamma T^{\beta}\right] F \frac{d F}{d \eta}-\left[\frac{T^{2 \beta}}{\Gamma} \frac{d \Gamma}{d T}\right] F=0
$$

For (32) being reduced to an expression in the single independent invariant $\eta$, it is necessary that the coefficients should be constants or functions of $\eta$ alone. Thus

$$
\begin{aligned}
& \beta X T^{\beta-1}=k_{1} \\
& \Gamma T^{\beta}=k_{2} \\
& \frac{T^{2 \beta}}{\Gamma} \frac{d \Gamma}{d \eta}=k_{3}
\end{aligned}
$$


Evaluation of $\beta$ and $\mathrm{k}_{3}$ gives us $\beta=0.5$ and $\mathrm{k}_{3}=-0.5$. hence from Eq.(35)

$$
\Gamma(T)=\frac{1}{\sqrt{T}}
$$

From which Eq. (32) takes the form

$$
2 \frac{d^{2} F}{d \eta^{2}}+(\eta-2 F) \frac{d F}{d \eta}+F=0
$$

Under the similarity variable $\eta$, the boundary conditions are transformed into

$$
\begin{aligned}
& F(0)=c_{0} \\
& F(\infty)=0
\end{aligned}
$$

\subsection{Analytical Solution of the Problem}

Eq. (36) takes the form

$$
\frac{d}{d \eta}\left(\frac{d F}{d \eta}-\frac{1}{2} F^{2}+\frac{1}{2} \eta F\right)=0
$$

Integrating (39), we get

$$
\frac{d F}{d \eta}-\frac{1}{2} F^{2}+\frac{1}{2} \eta F=k_{1} ; \text { where } k_{1} \text { is constant }
$$

Eq. (40) is a Riccati equation and its solution is obtained by considering two different cases.

Case-I: $\left\{\right.$ when $\left.F_{1}(\eta)=k_{2} \eta\right\}$

The Riccati equation (40) posses a special solution of the form

$$
F_{1}(\eta)=k_{2} \eta
$$

Which leads to $k_{1}=k_{2}$, and possible values of $k_{2}$ are 0 and 1 .

Reduction of Eq. (39) corresponding to $k_{2}=0$ from Eq. (41) is given by 


$$
\frac{d F}{d \eta}+\frac{1}{2} \eta F=\frac{1}{2} F^{2}
$$

Which is Bernoulli's equation and has the solution

$$
F(\eta)=\frac{e^{-\eta^{2} / 4}}{k_{3}-\frac{1}{2} \int_{0}^{\eta} e^{-\xi^{2} / 4} d \xi}
$$

Applying the condition (37), we get $k_{3}=\frac{1}{c_{0}}$.

Hence Eq. (39) has the solution

$$
F(\eta)=\frac{e^{-\eta^{2} / 4}}{\frac{1}{c_{0}}-\frac{1}{2} \int_{0}^{\eta} e^{-\xi^{2} / 4} d \xi}
$$

Which, can be rewritten as

$$
F(\eta)=\frac{e^{-\eta^{2} / 4}}{\frac{1}{c_{0}}-\left(\frac{\sqrt{\pi}}{2}\right) \operatorname{erf}\left(\frac{\eta}{2}\right)}
$$

and hence from Eq. (11), we get

$$
C(X, T)=\frac{1}{\sqrt{T}}\left[\frac{e^{-\eta^{2} / 4}}{\frac{1}{c_{0}}-(\sqrt{\pi} / 2) \operatorname{erf}\left(\frac{X}{2 \sqrt{T}}\right)}\right] ; X>0, T>0, c_{0} \neq 0 k_{2}=0 .
$$

Case-II: $\left\{\right.$ When $\left.F(\eta)=\eta+F_{2}(\eta)\right\}$

Reduction of Eq. (40) corresponding to $k_{2}=1$ and considering another special solution of Riccati equation (40) as, 


$$
F(\eta)=\eta+F_{2}(\eta)
$$

Hence Eq. (40) takes the form

$$
\frac{d F_{2}}{d \eta}+\frac{1}{2} \eta F_{2}=\frac{1}{2} F_{2}^{2}
$$

Again it is Bernoulli's equation in $F_{2}$ and $\eta$ and hence its solution is given by

$$
F_{2}(\eta)=\frac{e^{\eta^{2} / 4}}{k_{4}-\frac{1}{2} \int_{0}^{\eta} e^{-\xi^{2} / 4} d \xi}
$$

Hence from Eqs. (41), (42) and (49) we get

$$
F(\eta)=\eta+\frac{e^{\eta^{2} / 4}}{k_{4}-\frac{1}{2} \int_{0}^{\eta} e^{-\xi^{2} / 4} d \xi}
$$

Applying condition (37), we get $k_{4}=\frac{1}{c_{0}}$ and (38) is satisfied, hence we have

$$
F(\eta)=\eta+\frac{e^{-\eta^{2} / 4}}{\frac{1}{c_{0}}-\frac{1}{2} \int_{0}^{\eta} e^{-\xi^{2} / 4} d \xi}
$$

And hence the form of $\theta(Z, T)$ is given by,

$C(X, T)=\frac{X}{T}+\frac{1}{\sqrt{T}}\left[\frac{e^{X^{2} / 16 T}}{\frac{1}{c_{0}}-(\sqrt{\pi} / 2) \operatorname{erf}\left(\frac{X}{2 \sqrt{T}}\right)}\right] ; X>0, T>0, c_{0} \neq 0 k_{2}=1$.

\section{Interpretation of the Solution}

A specific problem of the longitudinal dispersion of miscible fluids flows through porous media under certain assumptions has been considered and its 


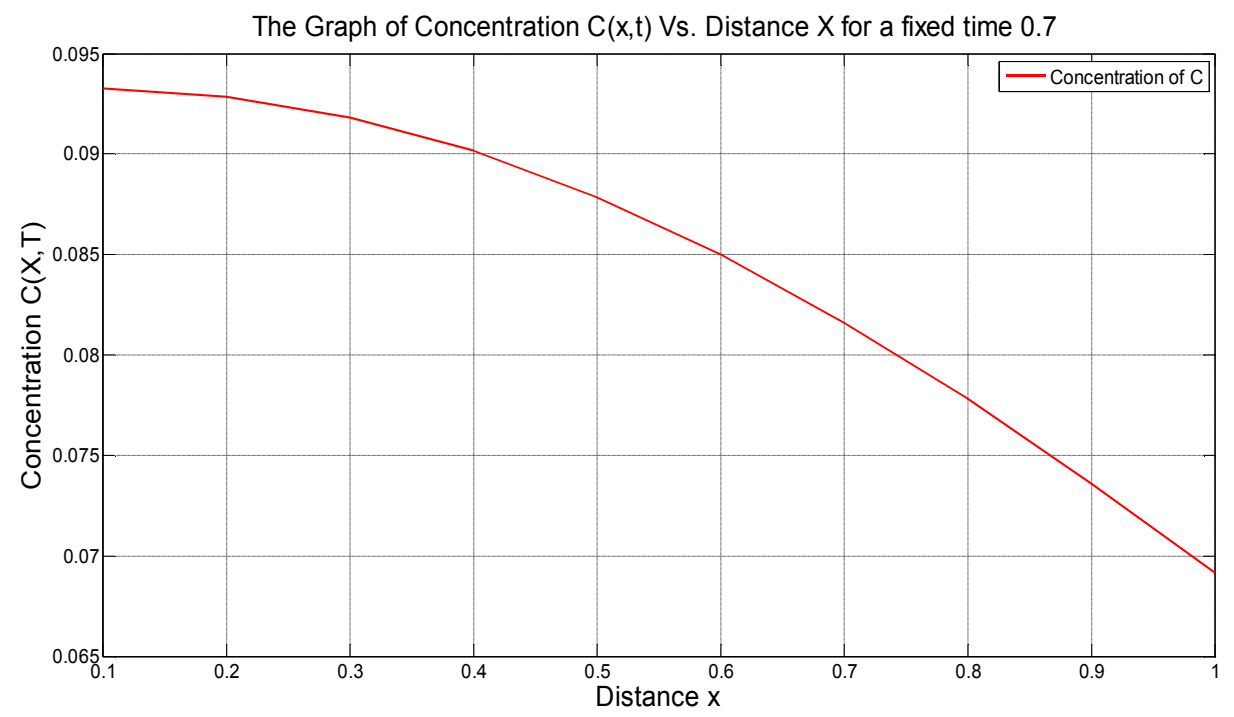

Figure 2 Concentration Vs. Distance for a fixed time $\mathrm{T}=0.7$.

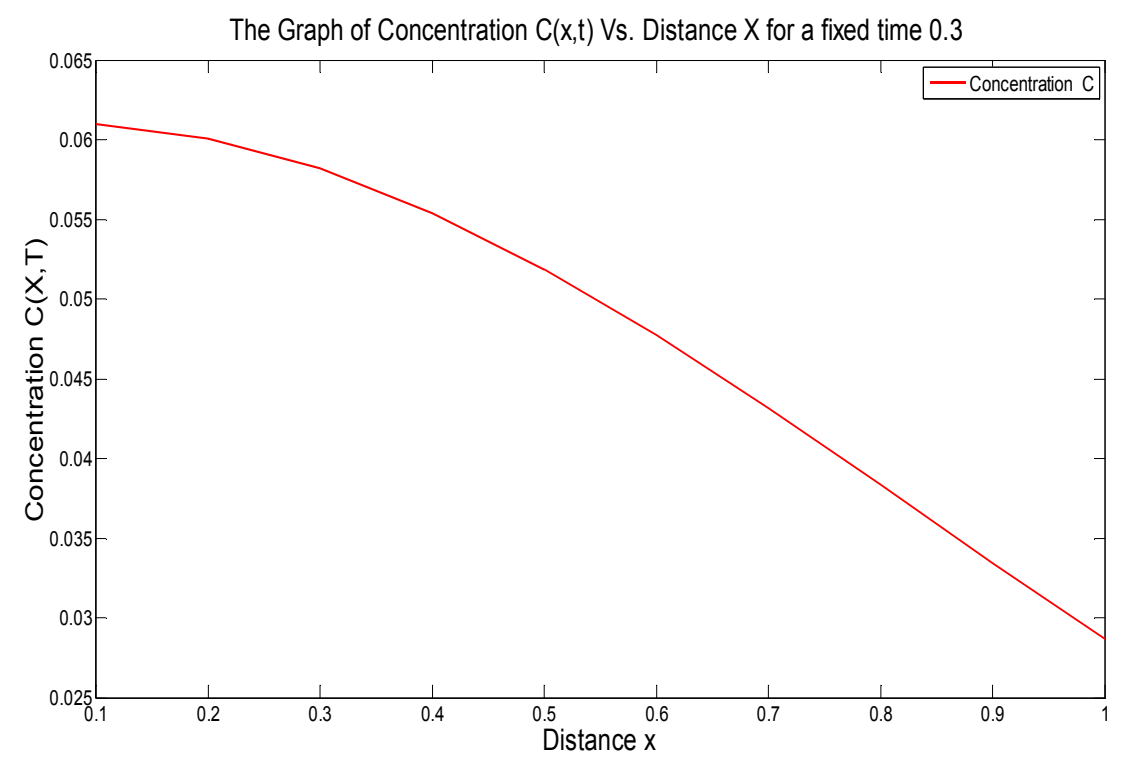

Figure 3 Concentration Vs. Distance for a fixed time $\mathrm{T}=0.3$.

solution is obtained by Group theoretic approach. We have obtained two different solutions as Eq. (46) and (52). Out of these two, the solution given by Eq. (46) is consistent with the physical situation as it can be observed form the 
solution (46) that the concentration in the longitudinal dispersion do have negative exponential term and hence concentration decreases with respect to distance $x$ for a fixed time $t>0$. Whereas Eq. (52), contains positive exponential term and hence concentration increases as the distance $x$ increases for a fixed time $t>0$. And hence we ignore the solution given by Eq. (52). The numerical and graphical representation has been obtained by using Mat lab programming. Fig. $2 \& 3$ represents the graph of concentration $\mathrm{C}$ vs. distance $\mathrm{X}$ for a fixed $\mathrm{T}=0.7 \& \mathrm{~T}=0.3$ respectively. From these two graphs we can conclude that concentration decreases as the distance $\mathrm{X}$ increases. Mehta and Saroj [12] have obtained the solution of this problem by taking different assumptions and by using the method of small parameters. Mehta \& Patel [11] have obtained the solution in the form of special functions.

\section{References}

[1] Ebach, E.H. \& White, R. Mixing of Fluids Flowing through Beds of Packed Solids, A. J. Ch. E., 4, p. 161, 1958.

[2] Ogta, A. \& Banks, R.B., A Solution of Differential Equation of Longitudinal Dispersion in Porous Media, Profess, Paper No. 411-A, 1961.

[3] Hoopes, J.A. \& Harteman, D.R.F., Waste Water Recharge and Dispersion in Porous Media, Technical Report No. 75, 1965.

[4] Bruce, J.C. \& Street, R.L., Studies of Free Surface Flow and Two Dimensional Dispersion in Porous Media, Report No. 63, Civil Eng. Dept. Stanford Uni., Stanford California, 1966.

[5] Marino, M.A., Flow Against Dispersion in Non Adsorbing Porous Media, J. Hydrology, 37, pp. 149-158, 1978.

[6] Al-Niami, A.N.S. \& Rushton, K.R., Analysis of Flow Against Dispersion in Porous Media, J. Hydrology., 33, pp. 87-97, 1977.

[7] Basak, P., Evaporation from Horizontal Soil Columns with Variable Diffusivity, J. Hydrology, 39, pp. 120-136, 1978.

[8] Hunt, B., Dispersion Calculations in Nonuniform Seepage, J. Hydrology, 36, pp. 261-277, 1978.

[9] Wang, St. T., McMillan, A.F. \& Chen B.H., Dispersion of Pollutants in Channels with Non Uniform Velocity Distribution, Water Research, 12, pp. 389-394, 1978.

[10] Kumar, N., Dispersion of Pollutants in Semi-Infinite Porous Media with Unsteady Velocity Distribution, Nordic Hydrology, pp. 167-178, 1983.

[11] Mehta, M.N. \& Patel, T.A., A Solution of Burger's Equation for Longitudinal Dispersion of Miscible Fluid Flow through Porous Media, Indian Journal of Petroleum Geology, 14(2), pp. 49-54, 2005. 
[12] Mehta, M.N. \& Saroj Yadav, Classical Solution of Non-Linear Equations Arising in Fluid Flow through Homogenous Porous Media, PhD thesis submitted to SVNIT, Surat, 2009.

[13] Bear, J., Dynamics of Fluids in Porous Media, Dover Publications, New York, 1972.

[14] Moran, M.J., Gaggioli, R.A., Reduction of The Number of Variables in System of Partial Differential Equations with Auxiliary Conditions, SIAM J. Appl. Math., 16, pp. 202-215, 1968. 